\title{
Challenges and advances in optimizing liver allografts from donation after circulatory death donors
}

\author{
Andrew lan Sutherland, \\ Gabriel C. Oniscu
}

Scottish Liver Transplant Unit, Royal Infirmary of Edinburgh, Edinburgh, UK

Address for correspondence:

Mr. Gabriel C. Oniscu, Transplant Unit, Royal Infirmary of Edinburgh, Little France Crescent,

Old Dalkeith Road, Edinburgh, EH16 4SA, UK. E-mail: gabriel.oniscu@ed.ac.uk

\begin{abstract}
In recent years, there has been a shift in the donor demographics with an increase in donation after circulatory death (DCD). Livers obtained from DCD donors are known to have poorer outcomes when compared to donors after brainstem death and currently only a small proportion of DCD livers are used. This review outlines the recent technological developments in liver DCD donation, including clinical studies using normothermic regional perfusion and extracorporal machine perfusion of livers from DCD donors.
\end{abstract}

Key words: Donors after circulatory death, liver transplantation, machine perfusion, normothermic regional perfusion, organ preservation

\section{INTRODUCTION}

Liver transplantation remains the only treatment for the end-stage liver disease. Despite advances in transplantation and an increase in the number of donors, there remains a significant disparity between organ availability and the clinical need. In recent years, there has been a shift in donor demographics with an increase in donation after circulatory death (DCD). DCD donation currently represents over $40 \%$ of all deceased donation in the $\mathrm{UK}^{[1]}$, and similar figures are seen in other European countries. ${ }^{[2]}$ In the United States, only 6-8\% of organs transplanted are from DCD donors, but this number is increasing. ${ }^{[3]}$ Whereas $87 \%$ of donors after brainstem death (DBD) livers are used, only $28 \%$ of livers recovered from DCD donors are transplanted in the $\mathrm{UK}^{[1]}$ [Figure 1]. Allografts obtained from DCD donors are known to have poorer outcomes

\begin{tabular}{|l|l|}
\hline \multicolumn{2}{|c|}{ Access this article online } \\
\hline Quick Response Code: & Website: \\
\cline { 1 - 2 } & www.jnsbm.org \\
\cline { 2 - 3 } & \\
\hline
\end{tabular}

when compared to brain dead organ donors. ${ }^{[4]}$ Prolonged exposure to ischemia, particularly warm ischemia followed by cold ischemia is known to exacerbate organ injury. ${ }^{[5]}$ Focus has therefore shifted within the transplant community to examine strategies for improving organ quality after DCD donation to allow more transplants to be performed with improved short- and long-term function. Interest has been reignited in machine perfusion, which allows an assessment of organ viability and a potential improvement of organ function. Machine perfusion of donor organs is not a new phenomenon. Huntley et al. first used in situ extracorporeal machine perfusion in 1963. ${ }^{[6]}$ However, these machines were expensive and difficult to transport, and with the advent of better and cheaper storage solutions such as Eurocollins and University of Wisconsin, static cold storage became the universal

This is an open access article distributed under the terms of the Creative Commons Attribution-NonCommercial-ShareAlike 3.0 License, which allows others to remix, tweak, and build upon the work non-commercially, as long as the author is credited and the new creations are licensed under the identical terms.

For reprints contact: reprints@medknow.com

How to cite this article: Sutherland Al, Oniscu GC. Challenges and advances in optimizing liver allografts from donation after circulatory death donors. J Nat Sc Biol Med 2016;7:10-5. 
preservation technique. As we look to expand the donor pool by using increasingly more extended criteria organs, particularly those from DCD donors, the use of machine perfusion is being revisited.

This review outlines the recent technological developments in liver DCD donation, including clinical studies using normothermic regional perfusion (NRP) and extracorporal machine perfusion of livers from DCD donors.

\section{THE CHALLENGES OF DONATION AFTER CIRCULATORY DEATH LIVER DONATION}

The number of DBD donors has declined in recent years. This is partly due to improved neurosurgical care and a reduction in the number of subarachnoid hemorrhages. ${ }^{[7]}$ DCD donors are, however, on the rise and have a significant contribution to the potential donor pool. ${ }^{[1]}$ DCDs are classified into four categories as part of the Maastricht criteria [Table 1]. The majority of donors worldwide are controlled DCD Category III donors. However, in Spain DCD Category II donors have been increasingly used, primarily with the introduction of NRP.

While DCD donors are a valuable source of organs, they are associated with poorer outcomes in liver transplantation. In renal transplantation, DCD kidneys have similar long-term outcomes to DBD kidneys, albeit with a higher incidence of delayed graft function. ${ }^{[3]}$ Liver transplantation, however, is associated with an increased incidence of primary nonfunction (3-7\%) and ischemic type biliary strictures (15-25\%). ${ }^{[8-10]}$ In addition up to $10 \%$ of the recipients die due to graft related causes with an additional $10 \%$ of patients being relisted for transplantation within a year of the initial transplant. The assessment of graft quality is notoriously difficult and relies heavily on surgeon's experience. As a result, only a small percentage of DCD livers are used [Figure 1].

Conventional organ preservation slows the metabolic processes to prolong the time during which an organ can be deprived of oxygen without loss of viability. However, despite slowing metabolism to a minimum, it does not halt it completely and subsequent damage results from adenosine triphosphate (ATP) depletion and mitochondrial damage. ${ }^{[11]}$ In organs procured from DCDs, there is an additional period of damaging warm ischemia from the time of the systolic blood pressure (BP) falling below 50 $\mathrm{mmHg}$ until the commencement of cold perfusion (known as functional warm ischemia). This ischemic process is distinct from cold ischemia damage and results in rapid cellular depletion of glycogen, oxygen, and ATP leading to anaerobic metabolism. The ATP depletion ultimately leads to failure of ATP dependent $\mathrm{Na}^{+} / \mathrm{K}^{+}$membrane pumps causing progressive cell swelling resulting in sinusoidal narrowing. ${ }^{[12,13]}$ This narrowing causes microcirculatory impedance and platelet and neutrophil aggregation. Furthermore, Kupffer cells become activated and release reactive oxygen species, which is aggravated on reperfusion. ${ }^{[14]}$

\section{TECHNOLOGICAL ADVANCES FOR DONATION AFTER CIRCULATORY DEATH LIVER OPTIMISATION}

Several technologies are currently explored for the recovery and preservation of DCD livers. Many offer the opportunity to assess organ viability prior to transplantation in order to allow a better selection of the livers grafts and minimize the incidence of graft failure and postoperative complications. Whilst the mechanisms of action for these novel technologies is yet to be fully understood, most aim at reversing the ATP depletion and minimize the degree of ischemia reperfusion injury. This also opens an exciting prospect for organ repair and reconditioning prior to

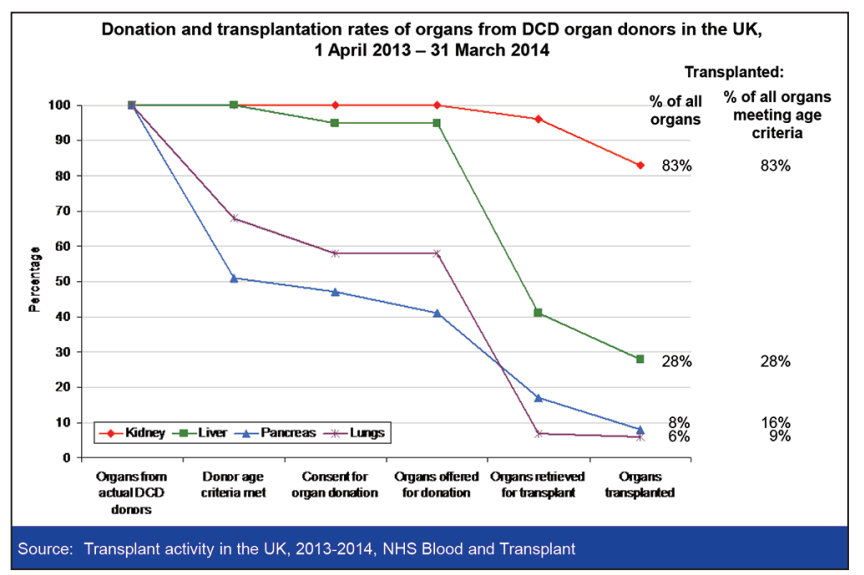

Figure 1: Organ utilization rates from donation after circulatory death donors in the UK

Table 1: Maastricht classification of donors after circulatory death

\begin{tabular}{llll}
\hline Category & Alternative categorization & Status of potential donor & Hospital department \\
\hline I & Uncontrolled & Dead upon arrival & Accident and emergency \\
II & Uncontrolled & Resuscitation attempted without success & Accident and emergency \\
III & Controlled & Awaiting cardiac arrest & Intensive care \\
IV & Controlled & Cardiac arrest while brain dead & Intensive care \\
\hline
\end{tabular}


transplantation, which may expand the pool of donor organs beyond the currently accepted criteria.

The field of liver optimization through novel organ recovery and preservation technologies is evolving rapidly, and several approaches have translated into clinical practice covering the entire pathway from organ recovery (in situ) to preservation and transport and perfusion prior to implantation (ex situ) at hypothermic, sub normothermic and normothermic temperatures. Given these different approaches there is a clear need for a uniform terminology to enable subsequent comparisons and a common approach for all transplanted organs.

\section{NORMOTHERMIC REGIONAL PERFUSION}

One potential way to improve the outcome of DCD liver transplantation is to restore the circulation with oxygenated blood to the abdominal organs in the donor in situ, using extracorporeal membrane oxygenation (ECMO) technology at body temperature. The use of ECMO to facilitate organ donation was first described by Johnson et al. in 1997. ${ }^{[15]}$ NRP, or normothermic recirculation, has been developed in Spain for uncontrolled DCD donors (Maastricht Category II). ${ }^{[16]}$ Following confirmation of death and a 5 min stand-off period, cardiorespiratory supportive maneuvers are restarted, and vascular access is obtained via the femoral vessels and a supraceliac balloon is inflated in the aorta to prevent brain reperfusion. The cannulae are connected to an extracorporeal circuit incorporating an oxygenator, centrifugal pump, a heat exchanger, and a reservoir. The donor blood is anticoagulated, and NRP is continued between 2 and 4 h. From 40 identified donors, the Barcelona group transplanted 10 livers, with one case of primary nonfunction and one hepatic artery thrombosis (HAT). The rate of ischemic type biliary strictures was $10 \%$, significantly lower than in many controlled DCD program, especially when considering that some of the donors had prolonged warm ischemia times. ${ }^{[16]}$ The same group subsequently reported an expanded cohort of 34 livers transplanted from DCD donors maintained with NRP, with the 1 -year recipient and graft survivals of $82 \%$ and $70 \%$, respectively (median follow-up 24 months). ${ }^{[17]}$

There have been a limited number of studies using normothermic extracorporeal support in controlled (Maastricht Category III) DCD donation. ${ }^{[18,19]}$ These reports suggested an apparent increase in the recovery rates of extra-renal organs with good initial function. In these studies, vascular cannulation and heparinization were performed prior to the declaration of death in order to minimize the risk of thrombosis post withdrawal of therapy. However, in some countries, including the UK, these interventions are not permitted. Our group has recently reported the current UK experience without prior heparinization. ${ }^{[20]}$ Following the declaration of death, a 5 min stand-off period was observed. Where possible, heparin was infused under pressure via existing cannulae while the patient was transferred to the operating room. The aorta and inferior vena cava were then cannulated following rapid midline laparotomy and after crossclamping of the aorta, NRP was commenced. The results from the first $21 \mathrm{NRP}$ retrievals in the UK have demonstrated that a period of $2 \mathrm{~h}$ of in situ NRP is safe and allows a more controlled pace of the donor procedure, thus minimizing the risk of organ damage. There was an increased organ recovery rate (mean of 3 organs/donor). In particular the liver utilization rate increased to $52 \%$ compared to in standard DCD donors, with no change in the current criteria for DCD selection, with a functional warm ischemia time $<30 \mathrm{~min}$ (WIT from systolic BP $<50 \mathrm{mmHg}$ to start of perfusion). Two hours of in situ NRP appeared to be sufficient to allow the assessment of liver graft function based on a composite index of macroscopic appearance, bile production, and dynamic biochemical markers analysis (serial measurements of lactate, base excess, bicarbonate, and alanine transaminase [ALT]). Posttransplant there was a lower mean ALT with a quicker trend to normalization during the $1^{\text {st }}$ week post liver transplant compared with standard DCD donors. No patients developed ischemic type biliary strictures during the follow-up (range 3-36 months), although one patient had primary nongraft function despite no worrying donor and perfusion characteristics. ${ }^{[20]}$

The use of NRP in DCD donors does not preclude the retrieval of DCD lungs, as dual temperature multiorgan retrieval is possible using abdominal NRP and concomitant cold lung flushing allowing both a rapid removal of the lungs and normothermic preservation of the abdominal organs. ${ }^{[21]}$

The use of in situ NRP is a significant advance in organ retrieval and has the potential to increase organ recovery rates due to its applicability in both controlled and uncontrolled DCD donors. Furthermore, the ability to restore the ATP supplies and the dynamic assessment of organ function prior to transplantation may allow a better selection of grafts and provide a superior long-term outcome compared to current DCD liver transplantation results. ${ }^{[22]}$

\section{HYPOTHERMIC MACHINE PERFUSION}

Whilst hypothermic machine perfusion (HMP) of kidneys is a part of routine clinical practice in many centers due to 
improved short-term outcomes, ${ }^{[23]}$ a similar approach to liver perfusion is in the early stages of development and clinical evaluation. Nonhuman studies have demonstrated the potential of $\mathrm{HMP}^{[24-27]}$ but to date, there have been very few clinical studies. Early experimental studies raised concerns that HMP may be associated with reduced bile flow, endothelial cell damage, and higher hepatic artery resistance ${ }^{[28,29]}$ potentially increasing the risk of HAT, however, recent clinical studies of HMP have been encouraging. Guarrera et al. reported the use of HMP in a prospective Phase 1 safety and feasibility study, comparing 20 recipients of HMP livers to a matched group of patients transplanted following static cold storage of the grafts. ${ }^{[30]}$ HMP was performed at the end of the cold storage period for 3-7 h using centrifugal perfusion with a customized solution (Vasosol) at $4-6^{\circ} \mathrm{C}$. There were no cases of primary nonfunction, and the HMP group exhibited less early allograft dysfunction rates (5\% vs. $25 \%$, although this did not reach significance). At 12 months, there were two deaths in each group, all unrelated to the preservation method. The recipients of HMP livers had fewer biliary complications and a shorter hospital stay; these findings were also confirmed in a subsequent report investigating the use of 31 extended criteria livers. ${ }^{[31]}$

Dutkowski et al. used a different approach employing hypothermic oxygenated perfusion (HOPE). ${ }^{[32]}$ The addition of oxygen was based on their previous experimental findings that oxygenated perfusion reduced endothelial cell and Kupffer cell activation and subsequent mitochondrial injury. ${ }^{[25,33]}$ In a pilot clinical study, 8 DCD (Maastricht Category III) livers were perfused and subsequently transplanted. ${ }^{[32]}$ Following asystole, a $10 \mathrm{~min}$ "no touch" period was observed prior to cannulating the iliac artery with a double balloon catheter and in situ flushing of the organs. The livers were retrieved with a mean functional warm ischemia time of $31 \mathrm{~min}$ and placed on the Extra-corporal Organ Perfusion System device (Organ Assist, Netherlands) at the transplant center. HOPE was performed at $10^{\circ}$ for $1-2 \mathrm{~h}$ prior to transplantation, whilst hepatectomy was undertaken in the recipient. In this small study, there was no primary nonfunction and liver transaminases were similar to a contemporaneous control group of DBD recipients. All recipients underwent magnetic resonance cholangiopancreatography with no evidence of cholangiopathy.

These clinical studies have demonstrated the safety and feasibility of HMP with or without the addition of oxygen. Other studies examined the use of HMP in discarded marginal livers and demonstrated that $4 \mathrm{~h}$ of HMP improves the markers of hepatocellular injury (aspartate transaminase and ALT) following rewarming compared to a cohort of cold-stored discarded livers. ${ }^{[34]}$
These early studies demonstrate the potential of HMP for organ resuscitation as supported by the analysis of the perfusate fluid. However, there still remain a number of unanswered questions, particularly with regards to the duration of perfusion, the optimal perfusion fluid and whether or not there is a need for active oxygenation.

\section{NORMOTHERMIC MACHINE PERFUSION}

Since the early conception of ex-vivo machine perfusion, there has been a cogent rationale for trying to maintain normal physiology when preserving organs. Ex-vivo normothermic machine perfusion (NMP) attempts to do this by mimicking the in vivo environment and perfusing organs with oxygenated blood at $37^{\circ} \mathrm{C}$. As with in situ NRP, this allows resuscitation of potential donor organs, as well as the clinical assessment of the appearance and biochemical evaluation of the function of the organ. Schön et al. demonstrated the potential value of NMP in an experimental large animal liver transplant model. ${ }^{[35]}$ Shortly after, Friend's group demonstrated livers maintain stable ex-vivo function following $72 \mathrm{~h}$ of NMP. ${ }^{[36]}$ The Oxford group went on to successfully transplant porcine livers after combinations of prolonged warm ischemia up to $40 \mathrm{~min}$ and preservation times of up to $20 \mathrm{~h} \cdot{ }^{\left[{ }^{37]}\right.}$ The same group has recently presented early clinical experience with 10 DBD livers which were successfully transplanted in adult recipients with the end-stage liver disease at Kings College Hospital in the UK. Although these were low-risk donors and recipients, the safety and feasibility of combining standard organ retrieval practice, back-bench preparation at the donor hospital and normothermic transportation and storage was demonstrated with 100\% graft and patient survival. ${ }^{[38]}$

An alternative strategy is a period of normothermic perfusion at the end of the cold storage period, prior to implantation, whilst recipient hepatectomy is undertaken. Porte's group used this approach in discarded livers and showed a beneficial effect on the liver recovery with the ability to assess the function of the graft. ${ }^{[3]]}$

Although this latter approach avoids the potential risks of graft loss in case of technology failure with normothermic storage and transport, it does not avoid organ cooling. Both small ${ }^{[40]}$ and large animal studies ${ }^{[5]}$ have demonstrated the detrimental effect of cooling, suggesting it may be desirable to eliminate cooling altogether. The Barcelona group combined NRP with NMP in a porcine model and showed that continuous normothermia achieves better results compared with any other storage and retrieval combination even after a prolonged warm ischemia time of $90 \mathrm{~min} \cdot{ }^{[4]}$

These experimental and clinical studies looked at a number of parameters to predict viability including transaminases, 
glucose metabolism, lactate, $\mathrm{pH}$, and bile production. However, it is still not clear, as with NRP, which are the best predictors of subsequent organ function. Porte's study suggested that bile output during perfusion may be the best parameter to predict viability. ${ }^{[39]}$ However, all these studies examine short-term markers indicating that these livers are unlikely to suffer primary nonfunction. However, ischemic cholangiopathy may not become apparent for up to a year after transplantation and, therefore, further long-term studies are required to determine the impact of these approaches on the development of this particular complication.

\section{FUTURE DEVELOPMENTS}

After over two decades of experimental research into machine perfusion and preservation in animal models, we are now seeing real progress in the clinical translation of these technologies. All three perfusion systems: HMP, NRP, and NMP, have embarked on or have completed early Phase 1 clinical studies. Randomized controlled trials that compare these three technologies to cold static storage have commenced for NMP and HMP, and a randomized controlled trial of NRP is planned for the near future.

These studies will allow us to determine the therapeutic effect of these technologies versus cold-static storage, whilst future studies may directly compare different technologies or their combined use. As our clinical experience with these technologies increases and there is a greater utilization of extended criteria organs, there is a need to determine the parameters that are best able to predict organ function and identify pharmacological interventions that used in combination with the novel technologies would allow us to recondition these organs. It is yet unclear if HMP, NMP, or NRP should be used alone or in combination or indeed if their use should be extended to all liver grafts.

Certainly, with the recent increase in DCD liver transplantation and its associated morbidity, one cannot afford to ignore the application of new technologies to assess, resuscitate and possibly treat donor's livers.

\section{Financial support and sponsorship}

Nil.

\section{Conflicts of interest}

There are no conflicts of interest.

\section{REFERENCES}

1. NHSBT. Organ Donation and Transplantation; 2014. p. 1-163. Available from: http://www.organdonation.nhs.uk/statistics/ transplant_activity_report/. [Last accessed on 2015 Sep 1].
2. Domínguez-Gil B, Haase-Kromwijk B, Van Leiden H, Neuberger J, Coene L, Morel P, et al. Current situation of donation after circulatory death in European countries. Transpl Int 2011;24:676-86.

3. Bernat JL, D'Alessandro AM, Port FK, Bleck TP, Heard SO, Medina J, et al. Report of a National Conference on Donation after cardiac death. Am J Transplant 2006;6:281-91.

4. Casavilla A, Ramirez C, Shapiro R, Nghiem D, Miracle K, Bronsther $\mathrm{O}$, et al. Experience with liver and kidney allografts from non-heart-beating donors. Transplantation 1995;59:197-203.

5. Reddy SP, Bhattacharjya S, Maniakin N, Greenwood J, Guerreiro D, Hughes D, et al. Preservation of porcine non-heart-beating donor livers by sequential cold storage and warm perfusion. Transplantation 2004;77:1328-32.

6. Marchioro TL, Huntley RT, Waddell WR, Starzl TE. The use of extracorporeal perfusion for obtaining post mortem grafts. Surgery 1963;54:900-11.

7. Neugebauer H, Heuschmann PU, Jüttler E. DEcompressive Surgery for the treatment of malignant INfarction of the middle cerebral arterY - Registry (DESTINY-R): Design and protocols. BMC Neurol 2012;12:115.

8. Jay CL, Lyuksemburg V, Ladner DP, Wang E, Caicedo JC, Holl JL, et al. Ischemic cholangiopathy after controlled donation after cardiac death liver transplantation: A meta-analysis. Ann Surg 2011;253:259-64.

9. Fujita S, Mizuno S, Fujikawa T, Reed AI, Kim RD, Howard RJ, et al. Liver transplantation from donation after cardiac death: A single center experience. Transplantation 2007;84:46-9.

10. Manzarbeitia CY, Ortiz JA, Jeon H, Rothstein KD, Martinez O, Araya VR, et al. Long-term outcome of controlled, non-heart-beating donor liver transplantation. Transplantation 2004;78:211-5.

11. Vajdová K, Graf R, Clavien PA. ATP-supplies in the cold-preserved liver: A long-neglected factor of organ viability. Hepatology 2002;36:1543-52.

12. Selzner M, Selzner N, Jochum W, Graf R, Clavien PA. Increased ischemic injury in old mouse liver: An ATP-dependent mechanism. Liver Transpl 2007;13:382-90.

13. Huet PM, Nagaoka MR, Desbiens G, Tarrab E, Brault A, Bralet MP, et al. Sinusoidal endothelial cell and hepatocyte death following cold ischemia-warm reperfusion of the rat liver. Hepatology 2004;39:1110-9.

14. Montalvo-Jave EE, Escalante-Tattersfield T, Ortega-Salgado JA, Piña E, Geller DA. Factors in the pathophysiology of the liver ischemia-reperfusion injury. J Surg Res 2008;147:153-9.

15. Johnson LB, Plotkin JS, Howell CD, Njoku MJ, Kuo PC, Bartlett ST. Successful emergency transplantation of a liver allograft from a donor maintained on extracorporeal membrane oxygenation. Transplantation 1997;63:910-1.

16. Fondevila C, Hessheimer AJ, Ruiz A, Calatayud D, Ferrer J, Charco R, et al. Liver transplant using donors after unexpected cardiac death: Novel preservation protocol and acceptance criteria. Am J Transplant 2007;7:1849-55.

17. Fondevila C, Hessheimer AJ, Flores E, Ruiz A, Mestres N, Calatayud D, et al. Applicability and results of Maastricht type 2 donation after cardiac death liver transplantation. Am J Transplant 2012;12:162-70.

18. Valero R, Cabrer C, Oppenheimer F, Trias E, Sánchez-Ibáñez J, De Cabo FM, et al. Normothermic recirculation reduces primary graft dysfunction of kidneys obtained from non-heart-beating donors. Transpl Int 2000;13:303-10.

19. Magliocca JF, Magee JC, Rowe SA, Gravel MT, Chenault RH $2^{\text {nd }}$, Merion RM, et al. Extracorporeal support for organ donation after cardiac death effectively expands the donor pool. J Trauma 2005;58:1095-101.

20. Oniscu GC, Randle LV, Muiesan P, Butler AJ, Currie IS, Perera MT, et al. In situ normothermic regional perfusion for controlled donation after circulatory death - The United Kingdom experience. Am J Transplant 2014;14:2846-54.

21. Oniscu GC, Siddique A, Dark J. Dual temperature multi-organ recovery from a Maastricht category III donor after circulatory death. Am J Transplant 2014;14:2181-6.

22. Butler AJ, Randle LV, Watson CJ. Normothermic regional perfusion for donation after circulatory death without prior heparinization. Transplantation 2014;97:1272-8. 


\section{Sutherland and Oniscu: Optimizing liver allografts}

23. Moers C, Smits JM, Maathuis MH, Treckmann J, van Gelder F, Napieralski BP, et al. Machine perfusion or cold storage in deceaseddonor kidney transplantation. N Engl J Med 2009;360:7-19.

24. Dutkowski P, Furrer K, Tian Y, Graf R, Clavien PA. Novel shortterm hypothermic oxygenated perfusion (HOPE) system prevents injury in rat liver graft from non-heart beating donor. Ann Surg 2006;244:968-76.

25. Schlegel A, Graf R, Clavien PA, Dutkowski P. Hypothermic oxygenated perfusion (HOPE) protects from biliary injury in a rodent model of DCD liver transplantation. J Hepatol 2013;59:984-91.

26. Matsuno N, Uchiyama M, Iwamoto H, Hama K, Narumi Y, Kikuchi K, et al. Machine perfusion preservation for liver transplantation from non-heart-beating donors with agonal stage. Transplant Proc 2002;34:2610-1.

27. Uchiyama M, Matsuno N, Nakamura $Y$, Iwamoto H, Hama K, Narumi K, et al. Usefulness of preservation by machine perfusion of liver grafts from non-heart-beating donors-a porcine model. Transplant Proc 2003;35:105-6.

28. Jain S, Xu H, Duncan H, Jones JW Jr, Zhang JX, Clemens MG, et al. $E x$-vivo study of flow dynamics and endothelial cell structure during extended hypothermic machine perfusion preservation of livers. Cryobiology 2004;48:322-32.

29. 't Hart NA, der van Plaats A, Leuvenink HG, van Goor H, Wiersema-Buist J, Verkerke GJ, et al. Determination of an adequate perfusion pressure for continuous dual vessel hypothermic machine perfusion of the rat liver. Transpl Int 2007;20:343-52.

30. Guarrera JV, Henry SD, Samstein B, Odeh-Ramadan R, Kinkhabwala M, Goldstein MJ, et al. Hypothermic machine preservation in human liver transplantation: The first clinical series. Am J Transplant 2010;10:372-81.

31. Guarrera JV, Henry SD, Samstein B, Reznik E, Musat C, Lukose TI, et al. Hypothermic machine preservation facilitates successful transplantation of "orphan" extended criteria donor livers. Am J Transplant 2015;15:161-9.
32. Dutkowski P, Schlegel A, de Oliveira M, Müllhaupt B, Neff F, Clavien PA. HOPE for human liver grafts obtained from donors after cardiac death. J Hepatol 2014;60:765-72.

33. Schlegel A, Rougemont OD, Graf R, Clavien PA, Dutkowski P. Protective mechanisms of end-ischemic cold machine perfusion in DCD liver grafts. J Hepatol 2013;58:278-86.

34. Monbaliu D, Liu Q, Libbrecht L, De Vos R, Vekemans K, Debbaut C, et al. Preserving the morphology and evaluating the quality of liver grafts by hypothermic machine perfusion: A proof-of-concept study using discarded human livers. Liver Transpl 2012;18:1495-507.

35. Schön MR, Kollmar O, Wolf S, Schrem H, Matthes M, Akkoc N, et al. Liver transplantation after organ preservation with normothermic extracorporeal perfusion. Ann Surg 2001;233:114-23.

36. Butler AJ, Rees MA, Wight DG, Casey ND, Alexander G, White DJ, et al. Successful extracorporeal porcine liver perfusion for $72 \mathrm{hr}$ Transplantation 2002;73:1212-8.

37. Brockmann J, Reddy S, Coussios C, Pigott D, Guirriero D, Hughes D, et al. Normothermic perfusion: A new paradigm for organ preservation. Ann Surg 2009;250:1-6.

38. Ravikumar R, Friend PJ. Human Liver Transplantation Using Normothermic Machine Preservation. ILTS Joint International Congress. The ILTS $20^{\text {th }}$ Annual Congress. Liver Transplantation, 2014; 20: S1-S399.

39. op den Dries S, Karimian N, Sutton ME, Westerkamp AC, Nijsten MW, Gouw AS, et al. Ex vivo normothermic machine perfusion and viability testing of discarded human donor livers. Am J Transplant 2013;13:1327-35.

40. McKeown CM, Edwards V, Phillips MJ, Harvey PR, Petrunka CN, Strasberg SM. Sinusoidal lining cell damage: The critical injury in cold preservation of liver allografts in the rat. Transplantation 1988;46:178-91.

41. Fondevila C, Hessheimer AJ, Maathuis MH, Muñoz J, Taurá P, Calatayud D, et al. Superior preservation of DCD livers with continuous normothermic perfusion. Ann Surg 2011;254:1000-7. 